\title{
CONTROLADOR INTELIGENTE APLICADO EM UM SISTEMA DE PULVERIZAÇÃ O LOCALIZADA DE AGROQUÍMICOS USANDO INJEÇÃO DIRETA
}

\author{
Kleber R. Felizardo*, Heitor V. Mercaldi*, Vilma A. Oliveira*, Paulo E. Cruvinel ${ }^{\dagger}$ \\ * Universidade de São Paulo \\ Avenida Trabalhador São-carlense, 400, CEP 13566-590 \\ São Carlos, SP, Brasil \\ $\dagger$ Embrapa Instrumentação \\ Rua XV de Novembro, 1452, CEP 13560-970 \\ São Carlos, SP, Brasil
}

Emails: klerfe@sc.usp.br, heitor@usp.br, vilma@sc.usp.br, paulo.cruvinel@embrapa.br

\begin{abstract}
The variable rate applications with direct injection systems is an important tool in precision agriculture to prevent from weed infestation. The injection system technology allow multiple chemical application and additionally reduces the toxicological and environmental risks associated with the carrier-chemical mix preparation and discard. This paper presents an implementation of a fuzzy gain scheduling of proportionalintegral- derivative (PID) controller as well as its comparison with a PID controller to adjust the fow rate of the carrier-chemical mix. Practical results obtained with a test bench for sprayers have proven the efectiveness of the fuzzy gain scheduling of PID controller proposed.
\end{abstract}

Keywords_ Precision agriculture, PID fuzzy control, direct injection, sprayer, variable-rate.

\begin{abstract}
Resumo - A aplicação localizada de agroquímicos em taxas variadas usando sistemas de pulverização com injeção direta é uma importante ferramenta da agricultura de precisão no combate da infestação de plantas daninhas. Sistemas de injeção direta possibilitam o uso de diferentes agroquímicos em uma mesma aplicação, e adicionalmente reduzem os impactos toxicológico e ambiental relacionados com o preparo e descarte da mistura de agroquímico e água. Neste artigo é apresentado uma implementação de um controlador adaptativo do tipo proporcional-integral-derivativo (PID) fuzzy por escalonamento de ganho bem como sua comparação a um controlador PID para ajustar a vazão da mistura água-agroquímico. Resultados práticos obtidos através de uma bancada de testes para pulverizadores comprovaram a eficiência do controlador PID fuzzy proposto.
\end{abstract}

Palavras-chave - Agricultura de precisão, controle PID fuzzy, injeção direta, pulverizador, taxa variada.

\section{Introdução}

A aplicação localizada de agroquímicos, realizada em taxa variável pela detecção instantânea dos alvos (por exemplo, plantas daninhas) através do uso de sensores ou câmeras digitais ou por mapas de prescrição, pode reduzir o desperdício de agroquímicos e a agressão ao meio ambiente, proporcionando uma produção mais eficiente de alimentos em grande escala e aumentando a produtividade agrícola. Na aplicação baseada em mapas, é necessário o mapeamento das infestações das plantas daninhas em uma etapa anterior a pulverização, para geração dos mapas de prescrição contendo os valores das doses do agroquímico para cada local do campo. No outro tipo de aplicação, a pulverização é feita em tempo real, ou seja, instantes após a identificação das plantas daninhas (Baio and Antuniassi, 2011).

As aplicações em taxa variada podem ser obtidas por mudanças do volume aplicado, mantendose a concentração da calda constante, ou através de variações na concentração (dose) do agroquímico na calda, ao longo da pulverização. Esse último método de variação é utilizado nos pulverizadores mais avançados que utilizam sistemas de injeção direta (Antuniassi and Júnior, 2000).

A principal característica dos sistemas de in- jeção direta está relacionada ao armazenamento do diluente (água) e do agroquímico em recipientes separados. A mistura é realizada somente no momento da aplicação, através da injeção do agroquímico na tubulação que leva a calda aos bicos do pulverizador. Nesses sistemas, a quantidade de agroquímico injetado pode ser realizada, entre outras maneiras, através do controle da rotação das bombas injetoras de pistões ou peristálticas, as quais possuem rotação proporcional a vazão. As principais vantagens do sistema de injeção direta são a redução dos riscos envolvidos no preparo e descarte da calda e a possibilidade de aplicar mais de um tipo de agroquímico numa mesma aplicação (Baio and Antuniassi, 2011).

Os controladores eletrônicos utilizados em sistemas de pulverização com injeção direta devem possuir como característica de desempenho um erro de regime inferior a 5\%. Adicionalmente, os valores instantâneos das vazões não podem ultrapassar $10 \%$ do valor de referência, devendo estes retornarem aos valores desejados em uma margem de $5 \mathrm{~s}$ após o pulverizador ter sofrido variações nas condições de trabalho, causados por alterações na velocidade do pulverizador ou mudanças nas taxas de aplicação (Rietz et al., 1997).

Os controladores eletrônicos, em sua configuração básica, são conectados a sensores (va- 
zão, rotação e de velocidade) e atuadores (válvula de controle e bomba de injeção) para regulagem das vazões da calda e do agroquímico. Os tipos mais simples de controladores em malha fechada empregados em sistemas de pulverização com injeção direta baseiam-se na lei de controle PID (Shi et al., 2009; Aiwu et al., 2010; Aissaoui et al., 2011). Entretanto, um controlador PID pode não atingir um desempenho satisfatório ou necessitar de frequentes ajustes manuais devido as características não-lineares do processo a ser controlado (Chen and Pham, 2001). As válvulas de controle utilizadas em sistemas de pulverização são não-lineares (zona-morta, saturação), assim como o comportamento dinâmico da vazão da calda e da pressão de pulverização (Steward and Humburg, 2000; Felizardo et al., 2012).

Controladores PID fuzzy por escalonamento de ganho, que consiste em um controlador PID e um sistema fuzzy, responsável pela alteração dos ganhos dos parâmetros desse controlador, têm sido aplicados para melhorar o desempenho dos controladores PID utilizados em processos nãolineares (Chen and Pham, 2001).

Este trabalho apresenta a implementação de um controlador adaptativo do tipo PID fuzzy por escalonamento de ganho, bem como sua comparação a um controlador PID para controle da vazão da calda de um sistema de pulverização para aplicação de agroquímicos a taxa variada usando injeção direta.

\section{Descrição do sistema de pulverização com injeção direta}

O sistema de pulverização para aplicação de agroquímicos a taxa variada desenvolvido neste trabalho (Figura 1) faz parte de uma bancada automatizada instalada em uma infraestrutura compartilhada entre a Embrapa Instrumentação e o Laboratório de Controle da Escola de Engenharia de São Carlos, da Universidade de São Paulo (Cruvinel et al., 2011). Esta bancada permite, dentre outras coisas, desenvolver novos tipos de controladores para sistemas de pulverização e por isso sua automação foi feita por um sistema embarcado baseado em um controlador programável para automação (PAC), modelo cRIO-9073, da National Instruments.

O pulverizador trabalha com taxa de aplicação variada e emprega injeção direta de agroquímico. O agroquímico é injetado na linha de sucção da bomba de pulverização (Figura 1) com o auxílio uma bomba de injeção do tipo pistão acionada por um motor CC de $12 \mathrm{~V}$. A vazão do agroquímico $q_{h}$ é proporcional a velocidade angular $\omega_{h}$ do motor CC. Um controlador proporcional-integral (PI) regula $\omega_{h}$ através da tensão média aplicada ao motor CC.

A bomba de pulverização também é do tipo

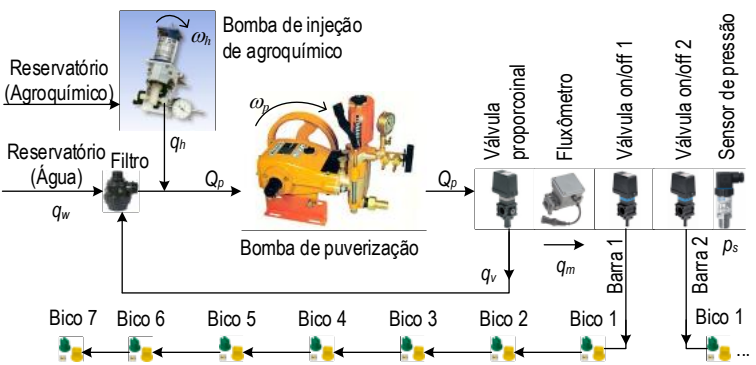

Figura 1: Ilustração do sistema de pulverização com injeção direta para aplicação de agroquímicos a taxa variada.

pistão acionado por um motor de indução trifásico e sua vazão $Q_{p}$ é proporcional a velocidade angular $\omega_{p}$ do motor de indução. O ajuste de $\omega_{p}$ é feito por um inversor de frequência e seu valor foi ajustado para manter a vazão da bomba em sua capacidade máxima.

Uma válvula de controle do tipo proporcional é acionada por um motor $\mathrm{CC}$ de $12 \mathrm{~V}$ e regula a vazão da mistura $q_{m}$, a qual é lida por um fluxômetro eletromagnético. O motor CC dessa válvula é acionado por uma ponte $\mathrm{H}$ comandada por um sinal do tipo PWM com frequência de $500 \mathrm{~Hz}$. O ciclo de trabalho desse sinal PWM, denotado por $d_{v}$, determina a ação de abertura, fechamento ou parada da válvula, e seu valor é ajustado pelo controlador adaptativo do tipo PID fuzzy por escalonamento de ganho.

As válvulas de seção de barra são do tipo on/off acionadas por motores CC de $12 \mathrm{~V}$ e são utilizadas para o fechamento das barras de pulverização 1 e 2. Nessas barras estão instalados os bicos de pulverização, com pontas de jato plano, com leque de $110^{\circ}$, modelo 11005 , e um sensor de pressão para monitoramento da pressão de pulverização $p_{s}$. Neste trabalho as válvulas de seção de barra permaneceram sempre abertas.

O PAC cRIO-9073 (Figura 2) possui um processador industrial de $266 \mathrm{MHz}$ gerenciado por um sistema operacional de tempo real, um chip FPGA (Field-Programmable Gate Array) de 2 milhões de portas programável pelo usuário e um chassi reconfigurável que aloja até oito módulos de entrada e/ou saída (E/S). O chip FPGA é conectado ao processador através de um barramento PCI de alta velocidade e cada módulo de E/S é conectado diretamente ao chip FPGA.

Para programar o cRIO-9073, utilizam-se os softwares LabVIEW PC, LabVIEW RT (RealTime) e LabVIEW FPGA. O processador do cRIO-9073 executa deterministicamente as aplicações criadas com o software LabVIEW RT e o chip FPGA executa de forma simultânea as aplicações criadas com o software LabVIEW FPGA. Neste projeto foi necessário desenvolver três programas (VIs). Dois programas foram criados no LabVIEW RT e LabVIEW FPGA os quais rodam 


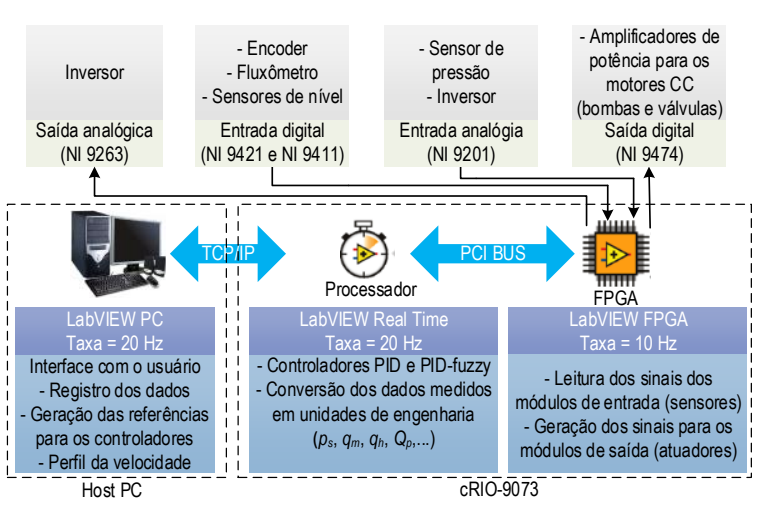

Figura 2: Arquitetura usada para o desenvolvimento da automação da bancada de pulverização.

diretamente no PAC. O outro programa foi criado no LabVIEW PC o qual é executado no PC Host (computador pessoal). As aplicações criadas nestes softwares estão descritas na Figura 2.

\section{Implementação dos controladores}

Neste trabalho são implementados e comparados dois controladores: um controlador PID convencional e um controlador PID fuzzy por escalonamento de ganho, que ajusta os parâmetros do PID através de um sistema fuzzy. Os controladores implementados para funcionamento no sistema de tempo real do cRIO-9073 foram desenvolvidos com o auxilio da ferramenta PID and Fuzzy Logic Toolkit do LabVIEW RT. A referência da vazão da calda $Q_{m r}$, em $\ell / \mathrm{min}$, para os controladores implementados é dada em função das seguintes variáveis (Boller and Raetano, 2011):

$$
Q_{m r}=\frac{D_{m r} v_{p} n e_{b}}{60000}
$$

sendo $D_{m r}(\ell / \mathrm{ha})$ a taxa de aplicação deseja para a calda, $v_{p}(\mathrm{~km} / \mathrm{h})$ a velocidade do pulverizador no campo, $e_{b}(\mathrm{~cm})$ a distância entre os bicos de pulverização e $n$ o número de bicos ativos na barra de pulverização.

\subsection{Controlador PID fuzzy com escalonamento de ganho}

Um controlador PID fuzzy com escalonamento de ganho foi proposto para regulagem da vazão da calda $q_{m}$. O controlador implementado é apresentado no diagrama em blocos da Figura 3.

O bloco de sintonia fuzzy, composto por quatro estágios detalhados na Figura 4, tem como objetivo ajustar os valores dos parâmetros do controlador PID para melhorar o desempenho da malha de controle. A escolha dos estágios 1 a 3 seguem as propostas apresentadas por $(\mathrm{Pu}$ and Zhang, 2009; Bae et al., 2011).

Estágio 1: é o estágio de entrada, responsável por

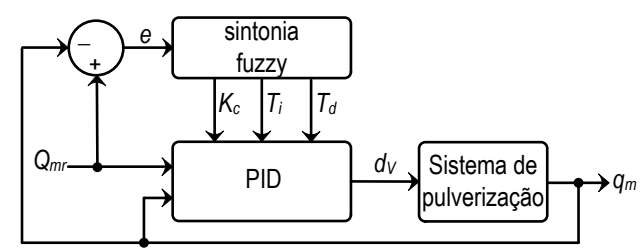

Figura 3: Diagrama em blocos do controlador PID fuzzy adaptativo implementado.

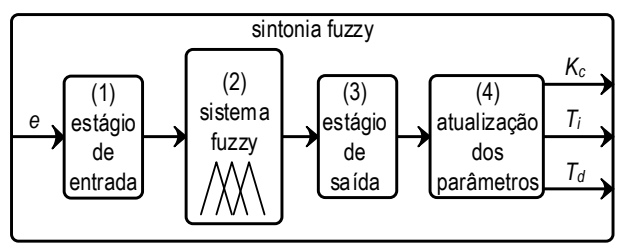

Figura 4: Diagrama em blocos do sistema de sintonia fuzzy.

ajustar os valores de entrada para o sistema fuzzy. Nesse estágio são calculados o erro $E$ e a variação do erro $E C$, variáveis de entrada do sistema fuzzy, como segue:

$$
\begin{gathered}
E=G_{E}\left(Q_{m r}-q_{m}\right)=G_{E} e(k T) \\
E C=G_{E C} \frac{e(k T)-e(k T-T)}{T}=G_{E C} \Delta e(k T)
\end{gathered}
$$

onde $G_{E}$ e $G_{E C}$ são as constantes de ganho e $T=50 \mathrm{~ms}$ é o período de amostragem.

Estágio 2: é o estágio onde ocorre a inferência para a sintonia do parâmetros. O sistema fuzzy é implementado com duas entradas e três saídas conforme ilustra a Figura 5.

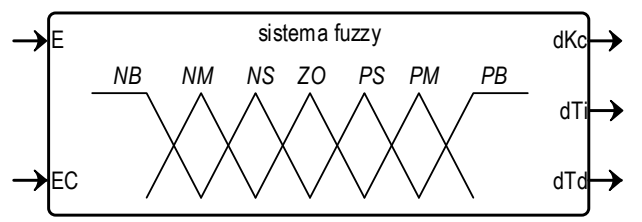

Figura 5: Representação do sistema fuzzy implementado.

A cada iteração são calculados os valores das saídas $d K_{c}, d T_{i}$ e $d T_{d}$ baseado no erro $E$ e sua variação $E C$. Cada variável, de entrada ou saída, é representada por um conjunto fuzzy composto por sete termos linguísticos: negativo grande (NB), negativo médio (NM), negativo pequeno (NS), zero (ZO), positivo pequeno (PS), positivo médio $(\mathrm{PM})$ e positivo grande (PB). Na Tabela 1 são mostrados os conjuntos fuzzy que representam as variáveis de entrada e saída, seu universo de discurso $\mathcal{U}$ e as variáveis linguísticas pertencentes a cada conjunto. A seguir são apresentadas em (4) a (6) as funções de pertinência que representam 
Tabela 1: Conjuntos fuzzy de entrada e saída.

\begin{tabular}{c|lcc}
\hline & Nome & $\mathcal{U}$ & Funções de pertinência \\
\hline variáveis & E & $(-41 ; 41)$ & $\{\mathrm{NB}, \mathrm{NM}, \mathrm{NS}, \mathrm{ZO}, \mathrm{PS}, \mathrm{PM}, \mathrm{PB}\}$ \\
de entrada & $\mathrm{EC}$ & $(-150 ; 150)$ & $\{\mathrm{NB}, \mathrm{NM}, \mathrm{NS}, \mathrm{ZO}, \mathrm{PS}, \mathrm{PM}, \mathrm{PB}\}$ \\
\hline variáveis & $d K_{c}$ & $(-1 ; 1)$ & $\{\mathrm{NB}, \mathrm{NM}, \mathrm{NS}, \mathrm{ZO}, \mathrm{PS}, \mathrm{PM}, \mathrm{PB}\}$ \\
de & $d T_{i}$ & $(-1 ; 1)$ & $\{\mathrm{NB}, \mathrm{NM}, \mathrm{NS}, \mathrm{ZO}, \mathrm{PS}, \mathrm{PM}, \mathrm{PB}\}$ \\
saída & $d T_{d}$ & $(-1 ; 1)$ & $\{\mathrm{NB}, \mathrm{NM}, \mathrm{NS}, \mathrm{ZO}, \mathrm{PS}, \mathrm{PM}, \mathrm{PB}\}$ \\
\hline
\end{tabular}

as variáveis de entrada $E$ e $E C$ e as de saída $d K_{c}$, $d T_{i}$ e $d T_{d}$

$$
\begin{aligned}
& E= \begin{cases}N B, & \mu_{\Pi}(x ;-42 ;-42 ;-10 ;-7) \\
N M, & \mu_{\bigwedge}(x ;-10 ;-7 ;-4) \\
N S, & \mu_{\bigwedge}(x ;-7 ;-4 ;-2) \\
Z O, & \mu_{\bigwedge}(x ;-4 ; 0 ; 4) \\
P S, & \mu_{\bigwedge}(x ; 2 ; 4 ; 7) \\
P M, & \mu_{\bigwedge}(x ; 4 ; 7 ; 10) \\
P B, & \mu_{\Pi}(x ; 7 ; 10 ; 42 ; 42)\end{cases} \\
& E C= \begin{cases}N B, & \mu_{\Pi}(x ;-150 ;-150 ;-11 ;-7) \\
N M, & \mu_{\bigwedge}(x ;-11 ;-7 ;-3) \\
N S, & \mu_{\bigwedge}(x ;-5 ;-3 ;-1) \\
Z O, & \mu_{\bigwedge}(x ;-3 ; 0 ; 3) \\
P S, & \mu_{\bigwedge}(x ; 1 ; 3 ; 5) \\
P M, & \mu_{\bigwedge}(x ; 3 ; 7 ; 11) \\
P B, & \mu_{\Pi}(x ; 7 ; 11 ; 150 ; 150)\end{cases} \\
& \begin{array}{ll}
d K_{c} \\
d T_{i} \\
d T_{d}
\end{array}= \begin{cases}N B, & \mu_{\bigwedge}(x ;-2 ;-1 ;-0,66) \\
N M, & \mu_{\bigwedge}(x ;-1 ;-0,66 ;-0,33) \\
N S, & \mu_{\bigwedge}(x ;-0,66 ;-0,33 ; 0) \\
Z O, & \mu_{\bigwedge}(x ;-0,33 ; 0 ; 0,33) \\
P S, & \mu_{\bigwedge}(x ; 0 ; 0,33 ; 0,66) \\
P M, & \mu_{\bigwedge}(x ; 0,33 ; 0,66 ; 1) \\
P B, & \mu_{\bigwedge}(x ; 0,66 ; 1 ; 2)\end{cases}
\end{aligned}
$$

Com as variáveis de entrada e saída definidas é elaborado o conjunto de regras (Tabela 2) que relacionam as entradas com as saídas, onde é utilizado o conectivo $A N D$ (Minimum). Para cada par de valores de $E$ e $E C$, o sistema fuzzy determina o valor das saídas através do método de defuzzificação centro de área $C o A$. As variáveis de saída do sistema fuzzy podem ser representadas com uma função das entradas e das regras:

$$
\begin{aligned}
d K_{c} & =F_{1}(E, E C), \\
d T_{i} & =F_{2}(E, E C), \\
d T_{d} & =F_{3}(E, E C)
\end{aligned}
$$

onde $F_{1}$ a $F_{3}$ são funções que mapeiam as variáveis de saída, e além disto, as saídas só são válidas $\left(d K_{c} \neq 0, d T_{i} \neq 0\right.$ e $\left.d T_{d} \neq 0\right)$ se os valores das variáveis de entrada estiverem representadas

\begin{tabular}{|c|c|c|c|c|c|c|c|c|}
\hline EC & $\mathrm{E}$ & NB & NM & NS & $\mathrm{ZO}$ & $\mathrm{P}$ & PM & PB \\
\hline \multirow{3}{*}{ NB } & $K_{c}$ & B & $\mathrm{PM}$ & $\mathrm{ZO}$ & NB & $\mathrm{ZO}$ & NS & $\mathrm{PB}$ \\
\hline & $T_{i}$ & O & NM & $\mathrm{ZO}$ & $\mathrm{ZO}$ & $\mathrm{ZO}$ & PB & NB \\
\hline & $T_{d}$ & $\mathrm{O}$ & NM & $\mathrm{ZO}$ & $\mathrm{ZO}$ & $\mathrm{ZO}$ & $\mathrm{NM}$ & $\mathrm{PB}$ \\
\hline \multirow{3}{*}{ NM } & $K_{c}$ & $\mathrm{~B}$ & $\mathrm{PM}$ & $\mathrm{ZO}$ & $\mathrm{NM}$ & $\mathrm{ZO}$ & NS & $\mathrm{PB}$ \\
\hline & $T_{i}$ & B & NM & $\mathrm{ZO}$ & $\mathrm{ZO}$ & $\mathrm{ZO}$ & $\mathrm{PB}$ & $\mathrm{NB}$ \\
\hline & $T_{d}$ & B & NM & $\mathrm{ZO}$ & $\mathrm{ZO}$ & $\mathrm{ZO}$ & $\mathrm{NM}$ & $\mathrm{PB}$ \\
\hline \multirow{3}{*}{ NS } & $K_{c}$ & B & $\mathrm{PM}$ & $\mathrm{ZO}$ & PB & $\mathrm{ZO}$ & $\mathrm{NS}$ & $\mathrm{PB}$ \\
\hline & $T_{i}$ & B & NM & $\mathrm{ZO}$ & $\mathrm{ZO}$ & $\mathrm{ZO}$ & $\mathrm{PB}$ & $\mathrm{NB}$ \\
\hline & $T_{d}$ & PB & NM & $\mathrm{ZO}$ & $\mathrm{ZO}$ & $\mathrm{ZO}$ & $\mathrm{NM}$ & $\mathrm{PB}$ \\
\hline \multirow{3}{*}{$\mathrm{ZO}$} & $K_{c}$ & B & $\mathrm{PM}$ & $\mathrm{ZO}$ & $\mathrm{PM}$ & $\mathrm{ZO}$ & NS & $\mathrm{P}$ \\
\hline & $T_{i}$ & B & $\mathrm{NM}$ & $\mathrm{ZO}$ & $\mathrm{ZO}$ & $\mathrm{ZO}$ & PB & NB \\
\hline & $T_{d}$ & PB & NM & $\mathrm{ZO}$ & $\mathrm{ZO}$ & $\mathrm{ZO}$ & NM & $\mathrm{PB}$ \\
\hline \multirow{3}{*}{ PS } & $K_{c}$ & $\mathrm{~PB}$ & $\mathrm{PM}$ & $\mathrm{ZO}$ & $\mathrm{PB}$ & $\mathrm{ZO}$ & NS & $\overline{\mathrm{PB}}$ \\
\hline & $T_{i}$ & NB & NM & $\mathrm{ZO}$ & $\mathrm{ZO}$ & $\mathrm{ZO}$ & $\mathrm{PB}$ & $\mathrm{NB}$ \\
\hline & $T_{d}$ & $\mathrm{~PB}$ & $\mathrm{NM}$ & $\mathrm{ZO}$ & $\mathrm{ZO}$ & $\mathrm{ZO}$ & $\mathrm{NM}$ & $\mathrm{PB}$ \\
\hline \multirow{3}{*}{ PM } & $K_{c}$ & $\mathrm{~PB}$ & $\mathrm{PM}$ & $\mathrm{ZO}$ & $\mathrm{NM}$ & $\mathrm{ZO}$ & NS & $\mathrm{PB}$ \\
\hline & $T_{i}$ & $\mathrm{NB}$ & $\mathrm{NM}$ & $\mathrm{ZO}$ & $\mathrm{ZO}$ & $\mathrm{ZO}$ & $\mathrm{PB}$ & $\mathrm{NB}$ \\
\hline & $T_{d}$ & $\mathrm{~PB}$ & NM & $\mathrm{ZO}$ & $\mathrm{ZO}$ & $\mathrm{ZO}$ & $\mathrm{NM}$ & $\mathrm{PB}$ \\
\hline \multirow{3}{*}{$\mathrm{PB}$} & $K_{c}$ & $\mathrm{~PB}$ & $\mathrm{PM}$ & $\mathrm{ZO}$ & NB & $\mathrm{ZO}$ & NS & $\mathrm{PB}$ \\
\hline & $T_{i}$ & NB & NM & $\mathrm{ZO}$ & $\mathrm{ZO}$ & $\mathrm{ZO}$ & $\mathrm{PB}$ & $\mathrm{NB}$ \\
\hline & $T_{d}$ & $\mathrm{~PB}$ & NM & $\mathrm{ZO}$ & $\mathrm{ZO}$ & $\mathrm{ZO}$ & $\mathrm{NM}$ & $\mathrm{PB}$ \\
\hline
\end{tabular}
em seus respectivos universos de discurso $E \in \mathcal{U}_{E}$ e $E C \in \mathcal{U}_{E C}$. Essas superfícies são definidas no intervalo $(-1,1)$ e os valores de variação dos parâmetros do controlador PID são obtidos no estágio
Tabela 2: Base de regras.

seguinte, pela multiplicação das superfícies pelos respectivos valores escalares dos ganhos de saída.

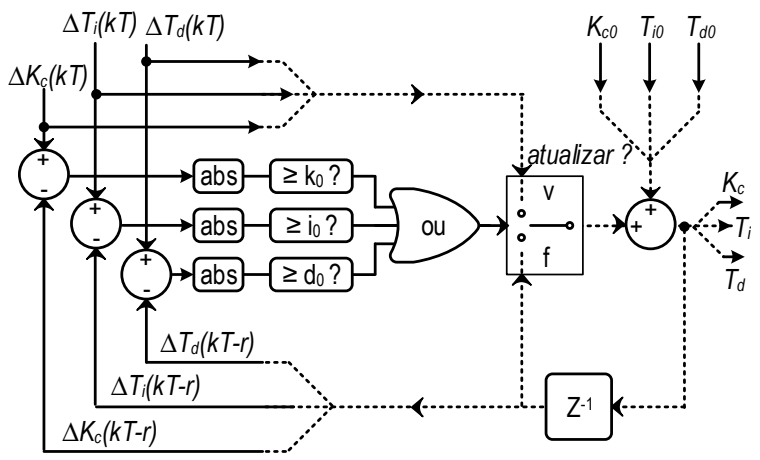

Figura 6: Esquema de avaliação e atualização dos parâmetros do controlador PID.

Estágio 3: é o estágio onde são calculados os valores da variação dos parâmetros do controlador PID. A partir das variáveis de saída do sistema fuzzy a variação dos parâmetros do controlador 
(a)

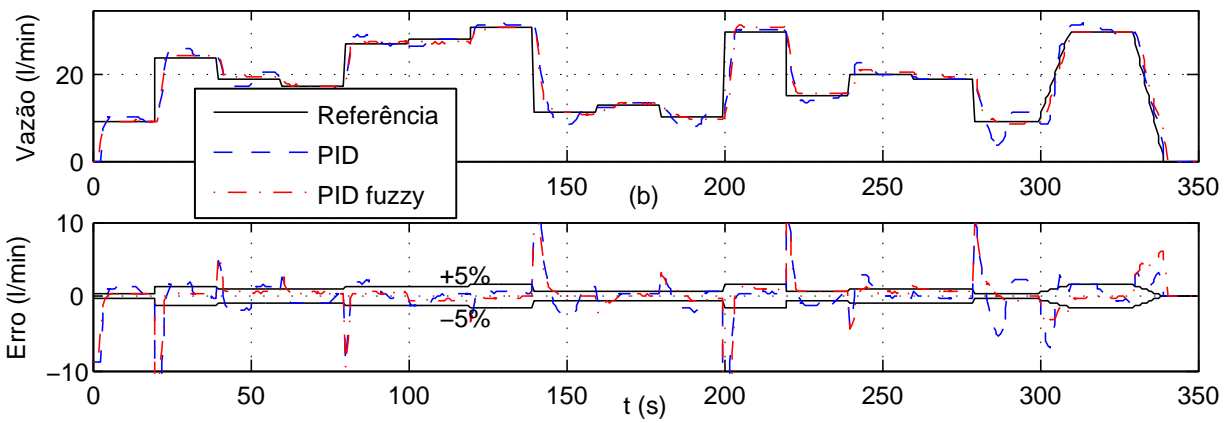

Figura 7: Respostas dos controladores PID e PID fuzzy.

PID é dada por:

$$
\begin{aligned}
\Delta K_{c}(k T) & =G_{K C} d K_{c}, \\
\Delta T_{i}(k T) & =G_{T I} d T_{i}, \\
\Delta T_{d}(k T) & =G_{T D} d T_{d},
\end{aligned}
$$

onde $G_{K C}=14, G_{T I}=3,510^{-2}$ e $G_{T D}=810^{-3}$ são ganhos do estágio e devem ser no máximo os valores ajustados no PID, a fim de evitar a instabilidade da malha de controle.

Estágio 4: é o estágio onde é verificado se os valores calculados do estágio anterior serão atualizados no controlador. A variação dos parâmetros $K_{c}, T_{i}$ ou $T_{d}$ durante a execução da rotina do PID acarreta na reinicialização do integrador, levando o controlador a atuar somente como PD. Para contornar esse problema é desenvolvido um algoritmo, representado através do esquema na Figura 6, que utiliza limiares para definir se atualiza ou não os parâmetros do controlador.

$\mathrm{O}$ algoritmo recebe como entradas os valores das variáveis de saída do sistema fuzzy $\left(\Delta K_{c}(k T)\right.$, $\Delta T_{i}(k T)$ e $\left.\Delta T_{d}(k T)\right)$, os valores dos ganhos aplicados as variáveis de saída do Estágio $3\left(G_{K C}\right.$, $G_{T I}$ e $\left.G_{T D}\right)$ e os valores dos parâmetros ajustados para o controlador PID $\left(K_{c 0}, T_{i 0}\right.$ e $\left.T_{d 0}\right)$ e verifica, para cada variável, se a diferença do valor absoluto entre as variáveis de saída são maiores que os limiares $\left(k 0=0,1 G_{K C}, i 0=0,1 G_{T I} \mathrm{e}\right.$ $\left.d 0=0,1 G_{T D}\right)$ e caso afirmativo, atualiza os valores dos parâmetros do controlador.

\subsection{Controlador PID}

Um controlador discreto do tipo PID foi utilizado para regulagem da vazão da calda $q_{m}$. Os parâmetros iniciais deste controlador, denotados pelo ganho proporcional $K_{c 1}$, pelo tempo de integração $T_{i 1}$ e pelo tempo de derivação $T_{d 1}$, foram obtidos a partir de ensaios na bancada de pulverização, utilizando-se o segundo método de Ziegler-Nichols (Ogata, 2003).

Inicialmente foi implementado um controlador proporcional. A saída $q_{m}$, para um ganho crítico $K_{c r}=20$, apresentou oscilações correspondente a um período crítico de $T_{c r}=3,2$. A partir de $K_{c r}$ e $T_{c r}$ foram encontrados os valores iniciais dos parâmetros $K_{c 1}, T_{i 1}$ e $T_{d 1}$. A partir dos valores iniciais obtidos pelo método, foi feito o ajuste manual dos parâmetros diretamente na bancada de pulverização e assim foram obtidos os valores dos parâmetros para o controlador PID do sistema de pulverização: $K_{c 1}=24$ e $T_{i 1}=0,044$ e $T_{d 1}=0,0067$, com $T_{i 1}$ e $T_{d 1}$ dado em minutos.

\section{Resultados}

O objetivo do ensaio apresentado a seguir é avaliar o desempenho dos controladores implementados, os quais são avaliados através do erro instantâneo da vazão $q_{m}$ no seguimento da trajetória de referência dada em (1). São analisados portanto o erro absoluto integral (IAE), dado em $\ell$, e a norma $\mathcal{L}^{2}$, dada em $\ell /$ min, que mede a raiz quadrática média do erro (Whitcomb et al., 1993; Oviedo et al., 2006).

É definida uma referência para a vazão da calda $Q_{m r}$, conforme mostra a Figura $7(\mathrm{a})$. O formato da curva utilizada como referência para os controladores foi elaborado para simular duas situações distintas: (i) mudanças abruptas na taxa de aplicação $D_{m r}$ devido a mudança do grau de infestação por plantas invasoras e (ii) variação na velocidade $v_{p}$ do pulverizador.

Os resultados experimentais mostrados na Figura 7 indicam que o controlador PID fuzzy apresentou um tempo de reposta mais rápido com menores sobressinais, bem como menores erros de vazão em regime. No geral, os gráficos da Figura 7 indicam um melhor desempenho do PID fuzzy, o que pode ser confirmado pelos índices de desempenho mostrados na Tabela 3.

Tabela 3: Desempenho dos controladores.

\begin{tabular}{ccc}
\hline Controlador & IAE & $\mathcal{L}^{2}$ \\
\hline PID & 9,060 & 2,990 \\
PID-Fuzzy & 7,280 & 2,880 \\
\hline Melhora (\%) & 25,00 & 4,00
\end{tabular}




\section{Conclusões}

Os resultados experimentais mostram que para regular a vazão da calda, o controlador PID fuzzy por escalonamento de ganho apresentou desempenho superior ao controlador PID convencional. Com relação ao erro total de aplicação houve uma melhora de $25 \%$ e quanto ao erro quadrático médio a melhora de desempenho é de $4 \%$ na faixa de operação de 10 a $32 \ell /$ min. Desta forma, concluise que o controlador PID fuzzy por escalonamento de ganho apresenta potencial aplicação para regular a vazão em sistemas de pulverização.

\section{Agradecimentos}

Os autores agradecem a Embrapa e a EESCUSP pela parceira no âmbito da Rede em Agricultura de Precisão (Macro Programa 1, Processo: 01.09.01.002.01) e ao CNPq (Processos: 143452/2008-8, 479306/2008-7 e 304985/2009-0).

\section{Referências}

Aissaoui, A. E., Lebeau, F., Elbahir, L., Destain, M.-F. and Houmy, K. (2011). A feasibility study of developing direct injection spraying technology for small scale farms, Synergy 2011, Godollo, Hungary, pp. 9-15.

Aiwu, C., Ailin, L., Zhizhuang, L., Wenzhao, Z. and Xinling, T. (2010). Design of flow control system on sprayer, International conference on computer application and system modeling, Taiyuan, pp. 577-580.

Antuniassi, U. R. and Júnior, C. D. G. (2000). Aplicação localizada de produtos fitossanitários, Simpósio de Agricultura de Precisão, Viçosa, pp. 181-202.

Bae, K., Park, J. W., Kim, C.-H. and Han, H.S. (2011). Fuzzy gain scheduling for magnetic levitation control, The 21st International Conference on Magnetically Levitated Systems and Linear Drives, Korea Institute of Machinery and Materials, Daejeon - Korea, pp. SOCP-01.

Baio, F. H. R. and Antuniassi, U. R. (2011). Sistemas de controle eletrônico e navegação para pulverizadores, in U. R. Antuniassi and W. Boller (eds), Tecnologia de Aplicação para Culturas Anuais, Aldeia Norte, Passo Fundo.

Boller, W. and Raetano, C. G. (2011). Bicos e pontas de pulverização de energia hidráulica, regulagens e calibração de pulverizadores de barras, in U. R. Antuniassi and W. Boller (eds), Tecnologia de Aplicação para Culturas Anuais, Aldeia Norte, Passo Fundo.
Chen, G. and Pham, T. T. (2001). Introduction to Fuzzy Sets, Fuzzy Logic, and Fuzzy Control Systems, CRC Press, Boca Raton, Florida.

Cruvinel, P. E., de Oliveira, V. A., Felizardo, K. R. and Mercaldi, H. V. (2011). Bancada automatizada para ensaios e desenvolvimento de pulverizadores de agrotóxicos, aplicadores de fertilizantes líquidos e maturadores em culturas agrícolas sob manejo baseado em agricultura de precisão, Agricultura de Precisão: um Novo Olhar, Embrapa Instrumentação, São Carlos, SP, pp. 96-100.

Felizardo, K. R., Mercaldi, H. V., Oliveira, V. A. and Cruvinel, P. E. (2012). Implementação de um controlador preditivo para uso em um sistema de injeção direta de herbicida, XIX Congresso Brasileiro de Automática, Campina Grande, pp. 3571-3576.

Ogata, K. (2003). Engenharia de Controle Moderno, 4 edn, Prentice Hall Brasil, São Paulo, Brasil.

Oviedo, J., Boelen, T. and van Overschee, P. (2006). Robust advanced PID control RaPID: PID tuning based on engineering specifications, Control Systems, IEEE 26(1): 1519. DOI: 10.1109/MCS.2006.1580148

$\mathrm{Pu}$, L. and Zhang, X. (2009). An intelligent fuzzy-pid control system for high-speed solenoid valve, Fourth International Conference on Innovative Computing, Information and Control (ICICIC), IEEE, Kaohsiung, pp. 504-507.

Rietz, S., Pályi, B., Ganzelmeier, H. and László, A. (1997). Performance of electronic controls for field sprayers, Journal of Agricultural Engineering Research 68(4): 399 - 407.

DOI: 10.1006/jaer.1997.0217

Shi, Y., Liang, A., Yuan, H., Zhang, C. and Li, J. (2009). Modeling and simulation for a variable sprayerrate system, in D. Li and C. Zhao (eds), Computer and Computing Technologies in Agriculture II, Volume 1, Vol. 293 of IFIP Advances in Information and Communication Technology, Springer US, Boston, pp. $573-584$.

Steward, B. L. and Humburg, D. S. (2000). Modeling the raven SCS-700 chemical injection system with carrier control with sprayer simulation, Transactions of the ASAE 43(2): 231-245.

DOI: $10.13031 / 2013.2698$

Whitcomb, L., Rizzi, A. and Koditschek, D. (1993). Comparative experiments with a new adaptive controller for robot arms, IEEE Transactions on Robotics and Automation $\quad \mathbf{9}(1)$ : $\quad 59-70 . \quad$ DOI: $10.1109 / 70.210795$ 